\title{
DYNAMIC BOUNDARY CONTROLS OF A ROTATING BODY-BEAM SYSTEM WITH TIME-VARYING ANGULAR VELOCITY
}

\author{
BOUMEDIÈNE CHENTOUF
}

Received 7 December 2003 and in revised form 24 February 2004

This paper deals with feedback stabilization of a flexible beam clamped at a rigid body and free at the other end. We assume that there is no damping and the rigid body rotates with a nonconstant angular velocity. To stabilize this system, we propose a feedback law which consists of a control torque applied on the rigid body and either a dynamic boundary control moment or a dynamic boundary control force or both of them applied at the free end of the beam. Then it is shown that the closed loop system is well posed and exponentially stable provided that the actuators, which generate the boundary controls, satisfy some classical assumptions and the angular velocity is smaller than a critical one.

\section{Introduction}

The aim of this paper is to study the stabilization of the system presented in Figure 1.1. This system, introduced in [2], consists of a disk (D) with an elastic beam (B) attached to its center and perpendicular to the disk plan (see Figure 1.1). The disk (D) rotates freely around its axis with a nonconstant angular velocity, and the motion of the beam (B) is confined to a plane perpendicular to the disk. Such systems arise in the study of large-scale flexible space structures and are well known as a rotating body-beam system.

To stabilize this system, we propose a feedback law composed of either a dynamic boundary control force or a dynamic boundary control moment (or both of them) applied at the free end of the beam while a control torque is present on the disk. With classical assumptions (see $[19,20]$ ) on the actuator which generates the boundary controls, we prove that for any given angular velocity smaller than a critical one, the beam vibrations are forced to decay exponentially to zero and the disk rotates with a desired angular velocity. This is important because exponential stability is a very desirable property for such structures. Additionally, this result permits, on one hand, to have a wide class of exponentially stabilizing controllers. On the other hand, the dynamic nature of the proposed boundary controls provides extra degrees of freedom in designing controllers 


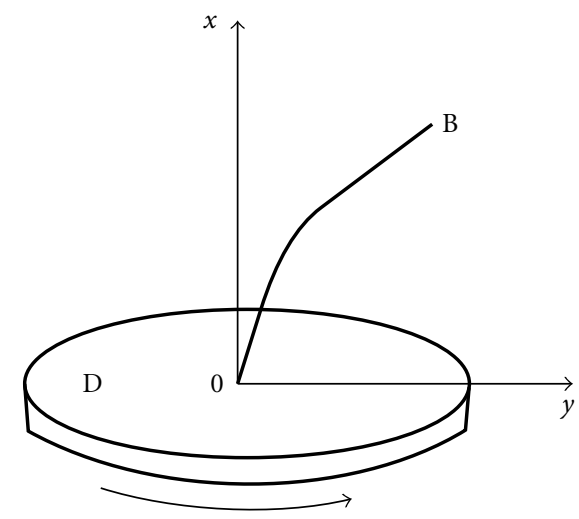

Figure 1.1. The body-beam system.

which could be exploited in solving control problems among which are pole assignment, disturbance rejection, and so on. From a practical viewpoint, one way of implementing the dynamic controls is to use gas jets at the tip of the beam and control the gas pressure by a dynamic actuator [19].

The global system is governed by the beam equation (PDE) nonlinearly coupled with the dynamical angular momentum equation (ODE) of the disk (D), that is,

$$
\begin{gathered}
\rho y_{t t}+E I y_{x x x x}=\rho \omega^{2}(t) y, \\
y(0, t)=y_{x}(0, t)=0, \\
E I y_{x x x}(l, t)=\alpha_{1} \Theta_{1}(t), \\
-E I y_{x x}(l, t)=\alpha_{2} \Theta_{2}(t), \\
\dot{\omega}(t)=\frac{\Theta_{3}(t)-2 \rho \omega(t)\left\langle y, y_{t}\right\rangle_{L^{2}(0, l)}}{I_{d}+\rho\|y\|_{L^{2}(0, l)}^{2}},
\end{gathered}
$$

where the positive constants $l, E I, \rho$, and $I_{d}$ are, respectively, the length of the beam, the flexural rigidity, the mass per unit length of the beam, and the disk's moment of inertia; where $\omega(t)$ is the angular velocity of the disk at time $t$, while $y(\cdot, t)$ is the beam's displacement in the rotating plane at time $t$. Moreover, $\alpha_{1}$ and $\alpha_{2}$ are two nonnegative constants such that

$$
\alpha_{1}+\alpha_{2} \neq 0
$$

and $\Theta_{1}(t), \Theta_{2}(t)$, and $\Theta_{3}(t)$ are, respectively, the control force, the control moment, and the control torque to be determined so that the solution's energy of the resulting closed loop system decays to zero in a suitable functional space.

The stabilization problem of the body-beam system has been extensively studied in the literature. In [2], the authors showed that with structural damping and without control, 
the body-beam system has a finite number of rotating equilibrium states. Later, Bloch and Titi [3] showed that in the more difficult case of viscous damping, a linear inertial manifold exists for the body-beam system. By taking into account the effect of damping, and for any constant angular velocity smaller than a critical one, an exponentially stabilizing feedback torque control law has been given in [24]. In the same case, and by adding a boundary force control, the system is also stabilizable for any constant angular velocity [25]. The stabilization problem of similar systems has been studied in $[17,18,20]$. For instance, in [20], the author considered a linear rotating body-beam subsystem, which is a reduced model of (1.1), by assuming that the angular velocity of the disk is constant, and thus the angular momentum equation of (1.1) is omitted. In this case, the author proposed dynamic boundary controls at the free end of the beam to obtain an exponential stabilization result. However, the presence of a force control was there necessary to achieve exponential stability. Later, for the body-beam system without damping, exponential stabilization was established in [16] as soon as at least one of two boundary controls (force or moment) is present at the free end of the beam with, in addition, a control torque of the disk. Recently, it was shown in [9] that the body-beam system without damping can be asymptotically stabilized by only a nonlinear feedback torque control law. The last result on this subject was obtained in [7] where the authors propose a wide class of nonlinear controls to establish the exponential stability of the body-beam system.

The main contribution of this paper is to show that the body-beam system is exponentially stabilized by means of a control torque on the disk and dynamic boundary controls (force and/or moment) applied at the free end of the beam. To prove this main result, we first consider a decoupled subsystem and use LaSalle's principle together with Ingham's inequality [12] to show the strong stability of the subsystem. Next, the frequency domain method [11] and a compact perturbation result [22] are used to obtain the exponential stability of the subsystem. Finally, the exponential stability of the global system is shown. This generalizes earlier results due to [16, 20]. More precisely, in this work, the angular velocity of the disk is not assumed to be constant, contrary to [20]. In addition, we are able to conclude the exponential stabilization even if one only applies a dynamic control moment at the free end of the beam with of course a control torque on the disk. This is not the case in [20], since the presence of control force was impossible to circumvent for the exponential stability. Furthermore, the controls proposed in [16] (static feedback) can be obtained by deleting the actuator state in our dynamic controls. However, we forewarn the reader that as in [16], the decay rate, although exponential, is not uniform.

Now we briefly outline the content of this paper. In Section 2, we propose a dynamic feedback law satisfying classical hypotheses and we formulate the global closed loop system as a standard form of evolution equation. Next, we prove in Section 3 the existence and uniqueness of solutions for the global system. The key step is to show the wellposedness of a decoupled subsystem, and then we consider an appropriate Lyapunov function. Section 4, containing the essential part of the paper, is devoted to establishing the strong stability and uniform stability of the decoupled subsystem. Finally, we prove in Section 5 the main result, namely, the exponential stability of the global closed loop system. Our conclusions are given in Section 6. 


\section{Preliminaries and main result}

In order to stabilize system (1.1), we propose the following feedback control law as long as $\alpha_{i} \neq 0$ for $i=1,2$ :

$$
\begin{gathered}
\Theta_{i}(t)=c_{i}^{T} w_{i}(t)+d_{i} u_{i}(t), \quad i=1,2, \\
\dot{w}_{i}(t)=A_{i} w_{i}(t)+b_{i} u_{i}(t), \quad i=1,2, \\
\Theta_{3}(t)=-\gamma\left(\omega(t)-\omega_{*}\right), \quad \text { for each } \omega_{*} \in \mathbb{R},
\end{gathered}
$$

where $\gamma$ is a positive constant and, for $i=1,2, w_{i} \in \mathbb{R}^{n_{i}}$ is the actuator state, $A_{i} \in \mathbb{R}^{n_{i} \times n_{i}}$ is a constant matrix, $b_{i}, c_{i} \in \mathbb{R}^{n_{i}}$ are constant column vectors, the superscript $T$ stands for the transpose, $d_{i} \in \mathbb{R}$ is a constant real number, and the input $u_{i}(t)$ is defined as

$$
u_{1}(t)=y_{t}(l, t), \quad u_{2}(t)=y_{x t}(l, t), \quad t \in \mathbb{R}^{+}
$$

Note that, for $i=1,2, \alpha_{i}=0$ in (1.1) means that the corresponding boundary control $\Theta_{i}(t)$ is not applied, and therefore the corresponding controller given by the first two equations of (2.1) is absent. It is also important to recall that we assume throughout this paper that $\alpha_{1}$ and $\alpha_{2}$ are two nonnegative constants such that $\alpha_{1}+\alpha_{2} \neq 0$, that is, at least one of the dynamic boundary controls in (2.1) is applied.

As in [20] (see also [19]), when $\alpha_{i} \neq 0, i=1,2$, the following hypotheses are assumed to be satisfied throughout this paper. For $i=1,2$,

(H.I) all eigenvalues of the matrix $A_{i}$ are in the open left half-plane,

(H.II) the triplet $\left(A_{i}, b_{i}, c_{i}\right)$ is both observable and controllable,

(H.III) $d_{i} \geq 0$; moreover, there exists a constant $\gamma_{i}$ such that $d_{i} \geq \gamma_{i} \geq 0$ and the transfer function

$$
G_{i}(s)=d_{i}+c_{i}^{T}\left(s I-A_{i}\right)^{-1} b_{i}
$$

satisfies

$$
\mathfrak{R}\left\{G_{i}(i \mu)\right\}>\gamma_{i}, \quad i=1,2, \mu \in \mathbb{R}
$$

where $\mathfrak{R}$ denotes the real part. Furthermore, when $d_{i}>0$, we assume $\gamma_{i}>0$ as well.

Remark 2.1. (1) Assumption (H.III) implies that the transfer function $G_{i}$ is a strictly positive real function for $i=1,2$. Now we will give a more explicit description of the transfer function $G_{i}(\cdot)$. Indeed, one can write $G_{i}(i \mu)=\mathfrak{R}(\mu)+i \mathfrak{J}(\mu)$, where $\mathfrak{J}$ denotes the imaginary part. Then, it follows immediately from (2.3) that for $\mu$ sufficiently large,

$$
\mathfrak{J}(\mu)=\mathscr{O}\left(\mu^{-1}\right)
$$


where for a function $J$ and for $\mu$ sufficiently large, we denote by $O(J(\mu))$ any function satisfying $\mathrm{O}(J(\mu)) \leq K J(\mu)$ for some positive constant $K$. Furthermore, combining (2.4) and (2.5) yields

$$
\mathfrak{R}(\mu)>\gamma_{i}, \quad \mathfrak{R}(\mu) \longrightarrow \gamma_{i} \quad \text { as } \mu \longrightarrow \infty .
$$

(2) Using the well-known Kalman-Yakubovich lemma, one can conclude that, given any symmetric positive definite matrix $Q_{i} \in \mathbb{R}^{n_{i} \times n_{i}}$, there exist a symmetric positive definite matrix $P_{i} \in \mathbb{R}^{n_{i} \times n_{i}}$ and a vector $q_{i} \in \mathbb{R}^{n_{i}}$ such that

$$
\begin{aligned}
A_{i}^{T} P i+P_{i} A_{i} & =-q_{i} q_{i}^{T}-\epsilon_{i} Q_{i}, \\
P_{i} b_{i}-\frac{c_{i}}{2} & =\sqrt{d_{i}-\gamma_{i}} q_{i},
\end{aligned}
$$

for $\epsilon_{i}>0$ sufficiently small [23].

We now turn to the formulation of the problem. Let

$$
H_{0}^{n}=\left\{f \in H^{n}(0, l) ; f(0)=f_{x}(0)=0\right\} \quad \text { for } n=2,3, \ldots,
$$

and let $\mathscr{X}$ be the state space, defined by

$$
\mathscr{X}=H_{0}^{2} \times L^{2}(0, l) \times \mathbb{R}^{n_{1}} \times \mathbb{R}^{n_{2}} \times \mathbb{R}=\mathscr{H} \times \mathbb{R},
$$

equipped with the following inner product:

$$
\begin{aligned}
& \left\langle\left(y, z, w_{1}, w_{2}, \omega\right),\left(\tilde{y}, \tilde{z}, \tilde{w}_{1}, \tilde{w}_{2}, \tilde{\omega}\right)\right\rangle \\
& \quad=\int_{0}^{l}\left(E I y_{x x} \tilde{y}_{x x}+\rho z \tilde{z}\right) d x+2 \sum_{i=1}^{i=2} \alpha_{i} \tilde{w}_{i}^{T} P_{i} w_{i}+\omega \tilde{\omega} .
\end{aligned}
$$

Note that the norm induced by this scalar product is equivalent to the usual one of the Hilbert space $H^{2}(0, l) \times L^{2}(0, l) \times \mathbb{R}^{n_{1}} \times \mathbb{R}^{n_{2}} \times \mathbb{R}$ by means of $(2.8)$ and the properties of the matrix $P_{i}, i=1,2$ (see part (2) of Remark 2.1). Next, setting $z(\cdot, t)=y_{t}(\cdot, t)$ and $\Phi(t)=\left(y(\cdot, t), z(\cdot, t), w_{1}(t), w_{2}(t), \omega(t)\right)$, the closed loop system (1.1)-(2.1)-(2.2) can be written into the following abstract form:

$$
\Phi_{t}(t)=\mathscr{A} \Phi(t)
$$

where $\mathscr{A}$ is an unbounded linear operator defined by

$$
\begin{aligned}
\mathscr{D}(\mathscr{A})=\{ & \Phi=\left(y, z, w_{1}, w_{2}, \omega\right) \in H_{0}^{4} \times H_{0}^{2} \times \mathbb{R}^{n_{1}} \times \mathbb{R}^{n_{2}} \times \mathbb{R} ; \\
& -E I y_{x x x}(l)+\alpha_{1}\left[c_{1}^{T} w_{1}+d_{1} z(l)\right]=0 ; \\
& \left.E I y_{x x}(l)+\alpha_{2}\left[c_{2}^{T} w_{2}+d_{2} z_{x}(l)\right]=0\right\},
\end{aligned}
$$

and for $\Phi \in \mathscr{D}(\mathscr{A})$,

$$
\mathscr{A} \Phi=\left(z,-\frac{E I}{\rho} y_{x x x x}+\omega_{*}^{2} y, A_{1} w_{1}+b_{1} z(l), A_{2} w_{2}+b_{2} z_{x}(l), 0\right)+\mathscr{B} \Phi,
$$


where $\mathscr{B}$ is a nonlinear operator in $\mathscr{X}$ defined by

$$
\mathscr{B} \Phi=\left(0,\left(\omega^{2}-\omega_{*}^{2}\right) y, 0,0, \frac{-\gamma\left(\omega-\omega_{*}\right)-2 \rho \omega\langle y, z\rangle_{L^{2}(0, l)}}{I_{d}+\rho\|y\|_{L^{2}(0, l)}^{2}}\right) \quad \forall \Phi \in \mathscr{X} .
$$

The main result of this paper is the following theorem.

Theorem 2.2. Assume that $d_{i}>0$ whenever the feedback gain $\alpha_{i}>0$, for $i=1,2$. Then, for each desired angular velocity $\omega_{*}$ satisfying $\left|\omega_{*}\right|<\left(1 / l^{2}\right) \sqrt{12 E I / \rho}$ and for each initial data $\Phi_{0} \in \mathscr{D}(\mathscr{A})$, the solution $\Phi(t)$ of (2.11) exponentially tends to the equilibrium point $\left(0 \mathscr{H}, \omega_{*}\right)$ in $\mathscr{X}$ as $t \rightarrow \infty$.

\section{Well-posedness of the problem}

In this section, we study the existence and uniqueness of the solutions of (2.11). First, consider the following subsystem in the space $\mathscr{H}=H_{0}^{2} \times L^{2}(0, l) \times \mathbb{R}^{n_{1}} \times \mathbb{R}^{n_{2}}$ :

$$
\phi_{t}(t)=A^{\omega_{*}} \phi(t), \quad \phi(0)=\phi_{0},
$$

where $A^{\omega_{*}}$ is an unbounded linear operator defined by

$$
\begin{aligned}
\mathscr{D}\left(A^{\omega_{*}}\right)=\{ & \phi=\left(y, z, w_{1}, w_{2}\right) \in H_{0}^{4} \times H_{0}^{2} \times \mathbb{R}^{n_{1}} \times \mathbb{R}^{n_{2}} ; \\
& -E I y_{x x x}(l)+\alpha_{1}\left[c_{1}^{T} w_{1}+d_{1} z(l)\right]=0 ; \\
& \left.E I y_{x x}(l)+\alpha_{2}\left[c_{2}^{T} w_{2}+d_{2} z_{x}(l)\right]=0\right\},
\end{aligned}
$$

and for $\phi \in \mathscr{D}\left(A^{\omega_{*}}\right)$,

$$
A^{\omega_{*}} \phi=\left(z,-\frac{E I}{\rho} y_{x x x x}+\omega_{*}^{2} y, A_{1} w_{1}+b_{1} z(l), A_{2} w_{2}+b_{2} z_{x}(l)\right) .
$$

One can claim that $\mathscr{H}=H_{0}^{2} \times L^{2}(0, l) \times \mathbb{R}^{n_{1}} \times \mathbb{R}^{n_{2}}$, endowed with the inner product

$$
\left\langle\left(y, z, w_{1}, w_{2}\right),\left(\tilde{y}, \tilde{z}, \tilde{w}_{1}, \tilde{w}_{2}\right)\right\rangle_{\mathscr{H}}=\int_{0}^{l}\left(E I y_{x x} \tilde{y}_{x x}-\rho \omega_{*}^{2} y \tilde{y}+\rho z \tilde{z}\right) d x+2 \sum_{i=1}^{i=2} \alpha_{i} \tilde{w}_{i}^{T} P_{i} w_{i},
$$

is a Hilbert space, provided that the assumption $\left|\omega_{*}\right|<\left(1 / l^{2}\right) \sqrt{12 E I / \rho}$ of Theorem 2.2 is satisfied. The following lemma concerns the well-posedness of system (3.1).

Lemma 3.1. Assume that $\left|\omega_{*}\right|<\left(1 / l^{2}\right) \sqrt{12 E I / \rho}$. Then

(i) the linear operator $A^{\omega_{*}}$, defined by (3.2)-(3.3), generates a $C_{0}$-semigroup of contractions $e^{t A^{\omega *}}$ on $\mathscr{H}=\overline{\mathscr{D}\left(A^{\omega_{*}}\right)}$,

(ii) for any initial data $\phi_{0} \in \mathscr{D}\left(A^{\omega_{*}}\right)$, system (3.1) admits a unique strong solution $\phi(t)=$ $e^{t A^{\omega_{*}}} \phi_{0} \in \mathscr{D}\left(A^{\omega_{*}}\right)$ for all $t \geq 0$ such that $\phi(\cdot) \in C^{1}\left(\mathbb{R}^{+} ; \mathscr{H}\right) \cap C\left(\mathbb{R}^{+} ; \mathscr{D}\left(A^{\omega_{*}}\right)\right) ;$ moreover, the function $t \mapsto\left\|A^{\omega_{*}} \phi(t)\right\|_{\mathscr{H}}$ is decreasing,

(iii) for any initial data $\phi_{0} \in \mathcal{H}$, system (3.1) has a unique weak solution $\phi(t)=e^{t A^{\omega *}} \phi_{0} \in$ $\mathscr{H}$ such that $\phi(\cdot) \in C^{0}\left(\mathbb{R}^{+} ; \mathscr{H}\right)$. 
Proof of Lemma 3.1. (i) Let $\phi=\left(y, z, w_{1}, w_{2}\right) \in \mathscr{D}\left(A^{\omega_{*}}\right)$. Using the inner product (3.4), one can obtain after a double integration by parts,

$$
\left\langle A^{\omega_{*}} \phi, \phi\right\rangle_{\mathscr{H}}=2 \sum_{i=1}^{i=2} \alpha_{i} w_{i}^{T} P_{i}\left(A_{i} w_{i}+b_{i} u_{i}(t)\right)-E I y_{x x x}(l) u_{1}(t)+E I y_{x x}(l) u_{2}(t)
$$

where $u_{i}(t)$ is given in (2.2). From the boundary conditions in (3.2) and the properties (2.7), it follows that

$$
\left\langle A^{\omega_{*}} \phi, \phi\right\rangle_{\mathscr{H}}=-\sum_{i=1}^{i=2} \alpha_{i}\left(\sqrt{d_{i}-\gamma_{i}} u_{i}(t)-w_{i}^{T} q_{i}\right)^{2}-\sum_{i=1}^{i=2} \alpha_{i} \gamma_{i} u_{i}^{2}(t)-\sum_{i=1}^{i=2} \alpha_{i} \epsilon_{i} w_{i}^{T} Q_{i} w_{i} .
$$

Therefore, the operator $A^{\omega_{*}}$ is dissipative. Next, using Lax-Milgram theorem [4], one can prove that $R\left(I-A^{\omega_{*}}\right)=\mathscr{H}$. Thus, Lumer-Phillips theorem implies that $A^{\omega_{*}}$ generates a $C_{0}$-semigroup of contractions $e^{t A^{\omega *}}$ on $\mathscr{H}=\overline{\mathscr{D}\left(A^{\omega_{*}}\right)}$.

Claims (ii) and (iii) are direct consequences of semigroups theory [4, page 105].

Now we are ready to deal with the global system (2.11).

Lemma 3.2. Assume that $\left|\omega_{*}\right|<\left(1 / l^{2}\right) \sqrt{12 E I / \rho}$. Then, for any initial data $\Phi_{0} \in \mathscr{X}$, the closed loop system (2.11) has a unique mild global bounded solution $\Phi(t) \in \mathscr{X}$. In return, if $\Phi_{0} \in \mathscr{D}(\mathscr{A})$, there exits a unique classical global solution $\Phi(t) \in \mathscr{D}(\mathscr{A})$.

Proof of Lemma 3.2. It is clear that the original system (2.11) can be written as follows:

$$
\left(\begin{array}{l}
\phi(t) \\
\omega(t)
\end{array}\right)_{t}=\left[\left(\begin{array}{cc}
A^{\omega_{*}} & 0 \\
0 & 0
\end{array}\right)+\mathscr{B}\right]\left(\begin{array}{c}
\phi(t) \\
\omega(t)
\end{array}\right)
$$

where $A^{\omega_{*}}$ and $\mathscr{B}$ are defined by (3.2)-(3.3) and (2.14), respectively. Since the linear operator $A^{\omega_{*}}$ generates a $C_{0}$-semigroup of contractions $e^{t A^{\omega *}}$ (see Lemma 3.1) and since $\mathscr{B}$ is continuously differentiable [24], it follows that for any $\Phi_{0}=\left(\phi_{0}, \omega_{0}\right) \in \mathscr{X}$, there is a unique local mild solution $\Phi(\cdot)=(\phi(\cdot), \omega(\cdot)) \in C([0, T] ; \mathscr{X})$ of $(3.7)$, for some $T>0$, given by the variation of constant formula [21]. We now show that this solution is global. To this end, we define the "energy" function

$$
\begin{aligned}
\mathscr{L}(\Phi)= & \sum_{i=1}^{i=2} \alpha_{i} w_{i}^{T} P_{i} w_{i}+\frac{1}{2} I_{d}\left(\omega-\omega_{*}\right)^{2}-\frac{1}{2} \omega_{*}^{2} \int_{0}^{l} \rho y^{2} d x \\
& +\frac{1}{2}\left(\omega-\omega_{*}\right)^{2} \int_{0}^{l} \rho y^{2} d x+\frac{1}{2} \int_{0}^{l}\left(\rho y_{t}^{2}+E I y_{x x}^{2}\right) d x .
\end{aligned}
$$

We claim that this function is a reasonable choice of Lyapunov function. Indeed, one can check that there exists a positive constant $K$ such that for all $\Phi \in \mathscr{X}$, we have $\mathscr{L}(\Phi) \geq$ $K\|\Phi\|_{\mathscr{Q}}^{2}$, provided that $\left|\omega_{*}\right|<\left(1 / l^{2}\right) \sqrt{12 E I / \rho}$. On the other hand, the regularity theorem [21] implies that each local solution of (3.7), with initial data in $\mathscr{D}(\mathscr{A})$, is a strong one. 
Moreover, a straightforward computation leads us to claim that for any initial condition $\Phi_{0} \in \mathscr{D}(\mathscr{A})$, the corresponding strong solution $\Phi$ of (3.7) satisfies

$$
\frac{d}{d t} \mathscr{L}(\Phi)=-\gamma\left(\omega-\omega_{*}\right)^{2}-E I y_{x x x}(l) u_{1}+E I y_{x x}(l) u_{2}+2 \sum_{i=1}^{i=2} \alpha_{i}\left\langle P_{i}\left(A_{i} w_{i}+b_{i} u_{i}\right), w_{i}\right\rangle
$$

where $u_{i}$ is given in (2.2). This, together with the boundary conditions of system $(2.11)$ and the properties (2.7), gives

$$
\frac{d}{d t} \mathscr{L}(\Phi)=-\gamma\left(\omega-\omega_{*}\right)^{2}-\sum_{i=1}^{i=2} \alpha_{i}\left(\sqrt{d_{i}-\gamma_{i}} u_{i}-w_{i}^{T} q_{i}\right)^{2}-\sum_{i=1}^{i=2} \alpha_{i} \gamma_{i} u_{i}^{2}-\sum_{i=1}^{i=2} \alpha_{i} \epsilon_{i} w_{i}^{T} Q_{i} w_{i}
$$

Consequently, $\mathscr{L}$ is a Lyapunov function. Hence, the solution of (2.11) stemmed from $\Phi_{0} \in \mathscr{D}(\mathscr{A})$ exists globally in a classical sense and is bounded. Finally, one can show that each weak solution exists globally and is bounded.

\section{Stability of the subsystem (3.1)}

In this section, we will show that the subsystem (3.1) is exponentially stable on $\mathcal{H}$. To do so, we first establish the strong stability.

4.1. Strong stability of $e^{t A^{\omega *}}$. Using LaSalle's invariance principle for infinite-dimensional systems [10], we will prove the strong stability of $e^{t A^{\omega *}}$. Note that this result has been obtained in [20] by means of the method of separation of variables. An alternative proof is given in this subsection by using Ingham's inequality [12]. First, using the compactness of the canonical embedding $i: \mathscr{D}\left(A^{\omega_{*}}\right) \rightarrow \mathscr{H}$ and the well-known result of Kato [13], one can readily show the following lemma.

Lemma 4.1. Assume that $\left|\omega_{*}\right|<\left(1 / l^{2}\right) \sqrt{12 E I / \rho}$,

(i) the operator $\left(A^{\omega_{*}}\right)^{-1}$ exists and is a compact one on $\mathscr{H}$,

(ii) the resolvent operator $\left(\lambda I-A^{\omega_{*}}\right)^{-1}: \mathscr{H} \rightarrow \mathcal{H}$ is compact for any $\lambda \geq 0$, and the spectrum of $A^{\omega_{*}}$ consists only of isolated eigenvalues with finite multiplicity.

We have the following proposition.

Proposition 4.2. Assume that $\left|\omega_{*}\right|<\left(1 / l^{2}\right) \sqrt{12 E I / \rho}$ and $d_{i}>0$ when $\alpha_{i}>0$ for $i=1,2$. The semigroup $e^{t A^{\omega *}}$ is strongly stable on $\mathscr{H}$, that is, for any initial condition $\phi_{0} \in \mathscr{H}$, the corresponding solution $\phi(t)=e^{t A^{\omega *}} \phi_{0}$ of (3.1) satisfies $\|\phi(t)\|_{\mathscr{H}} \rightarrow 0$ as $t \rightarrow+\infty$.

Proof of Proposition 4.2. By a standard argument of density of $\mathscr{D}\left(A^{\omega_{*}}\right)$ in $\mathscr{H}$ and the contraction of the semigroup $e^{t A^{\omega *}}$, it suffices to prove Proposition 4.2 for any initial data $\phi_{0} \in \mathscr{D}\left(A^{\omega_{*}}\right)$. Let $\phi(t)=e^{t A^{\omega *}} \phi_{0}$ be the solution of (3.1). It follows from Lemma 3.1(ii) that the trajectory of solution $\{\phi(t)\}_{t \geq 0}$ is a bounded set for the graph norm and thus precompact by virtue of Lemma 4.1(ii). Applying LaSalle's principle, we deduce that 
$\omega\left(\phi_{0}\right)$ is nonempty, compact, and invariant under the semigroup $e^{t A^{\omega *}}$, and, in addition, $e^{t A^{\omega *}} \phi_{0} \rightarrow \omega\left(\phi_{0}\right)$ as $t \rightarrow+\infty$ [10]. In order to prove the strong stability, it suffices to show that $\omega\left(\phi_{0}\right)$ reduces to zero. To this end, let $\tilde{\phi}_{0}=\left(\tilde{y}_{0}, \tilde{z}_{0}, \tilde{w}_{1_{0}}, \tilde{w}_{2_{0}}\right) \in \omega\left(\phi_{0}\right) \subset \mathscr{D}\left(A^{\omega_{*}}\right)$ and let $\tilde{\phi}(t)=\left(\tilde{y}(\cdot, t), \tilde{y}_{t}(\cdot, t), \tilde{w}_{1}(t), \tilde{w}_{2}(t)\right)=e^{t A^{\omega *}} \tilde{\phi}_{0} \in \mathscr{D}\left(A^{\omega_{*}}\right)$ be the unique strong solution of (3.1). We claim that $\tilde{\phi}(t)=0$, and therefore $\tilde{\phi}_{0}=0$. To see how this goes, recall that it is well known that $\|\tilde{\phi}(t)\|_{\mathscr{H}}$ is constant [10], and thus $(d / d t)\left(\|\tilde{\phi}(t)\|_{\mathscr{H}}^{2}\right)=0$, that is,

$$
\left\langle A^{\omega_{*}} \tilde{\phi}, \tilde{\phi}\right\rangle_{\mathscr{H}}=0 .
$$

Without loss of generality, we assume that $\alpha_{1}=0, \alpha_{2}>0$ (the case $\alpha_{2}=0, \alpha_{1}>0$ is similar). This implies, on one hand, that $u_{1}$ and $\tilde{w}_{1}$ are omitted and, on the other hand, $d_{2}, \gamma_{2}>0$ by means of the assumption of Proposition 4.2 and hypothesis (H.III). Combining (3.6) and (4.1), we deduce that $\tilde{w}_{2}=0$ and $\tilde{y}$ is a solution of the system

$$
\begin{gathered}
\rho \tilde{y}_{t t}+E I \tilde{y}_{x x x x}=\rho \omega_{*}^{2} \tilde{y}, \\
\tilde{y}(0, t)=\tilde{y}_{x}(0, t)=0, \\
\tilde{y}_{x x}(l, t)=\tilde{y}_{x x x}(l, t)=0, \\
\left(\tilde{y}(\cdot, 0), \tilde{y}_{t}(\cdot, 0)\right)=\left(\tilde{y}_{0}, \tilde{z}_{0}\right) \in H_{0}^{4} \times H_{0}^{2},
\end{gathered}
$$

with the additional condition

$$
\tilde{y}_{x t}(l, t)=0
$$

Obviously, to deduce the desired result $\tilde{\phi}(t)=0$, it suffices to show that $\tilde{y}=0$ is the only solution of (4.2)-(4.3). To do so, we will use the same techniques as in [8]. For simplicity, assume that $\rho=E I=l=1$. Then consider on the space $L^{2}(0,1)$ the operator $B_{0}$ defined by

$$
B_{0}=\frac{\partial^{4}}{\partial x^{4}}-\omega_{*}^{2} I, \quad \mathscr{D}\left(B_{0}\right)=\left\{f \in H^{4}(0,1) ; f(0)=f_{x}(0)=f_{x x}(1)=f_{x x x}(1)=0\right\} .
$$

It is easy to check that the operator $B_{0}$ is maximal, monotone, and selfadjoint with compact resolvent on $L^{2}(0,1)$. Hence, $B_{0}$ admits an infinity of real eigenvalues $0<\lambda_{1} \leq$ $\lambda_{2} \leq \cdots$, such that $\left(\lambda_{n}\right) \rightarrow+\infty$ as $n \rightarrow+\infty$ and the associated eigenfunctions $v_{1}, v_{2}, \ldots$ form an orthonormal basis of $L^{2}(0,1)$.

Now, we introduce a Hilbert space $\mathscr{H}_{*}=H_{0}^{2} \times L^{2}(0,1)$ with the inner product

$$
\langle(y, z),(\tilde{y}, \tilde{z})\rangle_{\mathscr{H}_{*}}=\int_{0}^{l}\left(y_{x x} \tilde{y}_{x x}-\omega_{*}^{2} y \tilde{y}+z \tilde{z}\right) d x .
$$

Next, consider the linear operator $A_{0}$ associated to system (4.2), namely, $A_{0}=\left(\begin{array}{cc}0 & I \\ -B_{0} & 0\end{array}\right)$ with $\mathscr{D}\left(A_{0}\right)=\mathscr{D}\left(B_{0}\right) \times H_{0}^{2}$. Clearly, the operator $A_{0}$ is skew-adjoint with compact resolvent on $\mathscr{H}_{*}$. Moreover, $\mu \in \sigma\left(A_{0}\right)$ if there exists a nontrivial $V=(y, z) \in \mathscr{D}\left(A_{0}\right)$ such that

$$
\begin{gathered}
B_{0} y=-\mu^{2} y, \quad y \in \mathscr{D}\left(B_{0}\right), \\
z=\mu y .
\end{gathered}
$$


Consequently, the eigenvalues $\mu_{n}$ and the associated eigenfunctions of $A_{0}$ can be deduced from those of $B_{0}$ as follows: $\mu_{n}= \pm i \sqrt{\lambda_{n}}$ and $V_{n}=\left(v_{n}, \pm i \sqrt{\lambda_{n}} v_{n}\right)$, for $n \in \mathbb{N}^{*}$. Observe that $\left\|V_{n}\right\|_{\mathscr{H}_{*}}^{2}=2 \lambda_{n}$ for any $n \in \mathbb{N}^{*}$. Therefore, in order to have an orthonormal basis of $\mathscr{H}_{*}$ and for convenience, we set $\mu_{n}=i \sqrt{\lambda_{n}}, \mu_{-n}=-i \sqrt{\lambda_{n}}$, for $n \in \mathbb{N}^{*}$, and $V_{n}=$ $\left(1 / \sqrt{2 \lambda_{n}}\right)\left(v_{n},-i \sqrt{\lambda_{n}} v_{n}\right), V_{-n}=\left(1 / \sqrt{2 \lambda_{n}}\right)\left(v_{n}, i \sqrt{\lambda_{n}} v_{n}\right)$. Obviously, the solution of $(4.2)$ is given by

$$
\left(\tilde{y}, \tilde{y}_{t}\right)(t)=\sum_{n \in \mathbb{Z}} C_{n} e^{-\mu_{n} t} V_{n}
$$

where $C_{n}=\left\langle\left(\tilde{y}_{0}, \tilde{z}_{0}\right), V_{n}\right\rangle_{\mathscr{H}_{*}}$ (for the complexified scalar product in $\left.(4.5)\right)$, for any $n \in \mathbb{Z}^{*}$. One finds that for $n \in \mathbb{N}^{*}, C_{n}=a_{n}+i b_{n}$ and $C_{-n}=a_{n}-i b_{n}$, where

$$
a_{n}=\frac{1}{\sqrt{2 \lambda_{n}}} \int_{0}^{1}\left(\tilde{y}_{0_{x x}} v_{n_{x x}}-\omega_{*}^{2} \tilde{y}_{0} v_{n}\right) d x, \quad b_{n}=\frac{1}{\sqrt{2}} \int_{0}^{1} \tilde{z}_{0} v_{n} d x .
$$

After an easy computation, we get from (4.7) and (4.8),

$$
\begin{aligned}
& \tilde{y}(t)=\sum_{n=1}^{\infty}\left(a_{n} \cos \left(\sqrt{\lambda_{n}} t\right)+b_{n} \sin \left(\sqrt{\lambda_{n}} t\right)\right) \frac{\sqrt{2}}{\sqrt{\lambda_{n}}} v_{n}, \\
& \tilde{y}_{t}(t)=\sum_{n=1}^{\infty}\left(-a_{n} \sin \left(\sqrt{\lambda_{n}} t\right)+b_{n} \cos \left(\sqrt{\lambda_{n}} t\right)\right) \sqrt{2} v_{n},
\end{aligned}
$$

where the series (4.9) and (4.10) converge in $H_{0}^{2}$ and $L^{2}(0,1)$, respectively, uniformly in $t$. Following the method used in [8], we will prove that $a_{n}=b_{n}=0$ for any $n=1,2, \ldots$, and thus $\left(\tilde{y}(t), \tilde{y}_{t}(t)\right)=(0,0)$. Indeed, $\left(\tilde{y}(0), \tilde{y}_{t}(0)\right)=\left(\tilde{y}_{0}, \tilde{z}_{0}\right)$ being in $H_{0}^{4} \times H_{0}^{2}$ (see (4.2)), one can claim that

$$
\tilde{y}_{0}=\tilde{y}(0)=\sqrt{2} \sum_{n=1}^{\infty} \frac{a_{n}}{\sqrt{\lambda_{n}}} v_{n} \in H_{0}^{2}, \quad \tilde{z}_{0}=\tilde{y}_{t}(0)=\sqrt{2} \sum_{n=1}^{\infty} b_{n} v_{n} \in H_{0}^{2} .
$$

Since $\left(v_{n} / \sqrt{\lambda_{n}}\right)_{n \geq 1}$ is an orthonormal basis for $H_{0}^{2}$, one can verify that the series defining $\tilde{y}_{t}(t)$ in (4.10) converges in $H_{0}^{2}$ uniformly in $t$. By continuity of the trace operator $u \mapsto$ $u_{x}(1)$ in $H_{0}^{2}$, (4.3) reads

$$
\tilde{y}_{x t}(1, t)=\sqrt{2} \sum_{n=1}^{\infty}\left(-a_{n} \sin \left(\sqrt{\lambda_{n}} t\right)+b_{n} \cos \left(\sqrt{\lambda_{n}} t\right)\right) v_{n_{x}}(1)=\sum_{n \in \mathbb{Z}} C_{n} e^{\mu_{n} t} v_{n_{x}}(1)=0 .
$$

Furthermore, the eigenvalues $\lambda_{n}$ and the eigenfunctions $v_{n}$ of $B_{0}$ satisfy the following properties (see the appendix for a proof):

$$
\lim _{n \rightarrow+\infty}\left|\sqrt{\lambda_{n+1}}-\sqrt{\lambda_{n}}\right|=\infty, \quad v_{n}(1) v_{n_{x}}(1) \neq 0, \quad n=1,2, \ldots
$$

Now, let $S_{N}(t)=\sum_{n=-N}^{n=N} C_{n} e^{\mu_{n} t} v_{n_{x}}(1), t>0$. We know from (4.12) that $\lim _{N \rightarrow+\infty} S_{N}(t)=0$ uniformly in $t \in[-T, T]$. Then, using Ingham's inequality [12], we deduce that there 
exists a constant $\kappa>0$ such that $\sum_{n=-N}^{n=N}\left|C_{n} v_{n_{x}}(1)\right|^{2} \leq \kappa \int_{-T}^{T}\left|S_{N}(t)\right|^{2} d t$. Therefore

$$
\sum_{n \in \mathbb{Z}}\left|C_{n} v_{n_{x}}(1)\right|^{2} \leq 0 \quad \text { as } N \longrightarrow+\infty
$$

This, together with (4.13), means that $C_{n}=0$ for any $n \in \mathbb{Z}$, and thus $\left(\tilde{y}, \tilde{y}_{t}\right)=0$. The proof of Proposition 4.2 is complete.

Remark 4.3. Obviously, the case $\alpha_{1} \alpha_{2}>0$ is a consequence of the case $\alpha_{1}=0, \alpha_{2}>0$ or $\alpha_{2}=0, \alpha_{1}>0$.

4.2. Exponential stability of $e^{t A^{\omega *}}$. The following technical result is crucial.

Theorem 4.4. Assume that $\left|\omega_{*}\right|<\left(1 / l^{2}\right) \sqrt{12 E I / \rho}$ and $d_{i}>0$ when $\alpha_{i}>0$ for $i=1,2$. Then, the semigroup $e^{t A^{\omega *}}$ is uniformly exponentially stable on $\mathscr{H}$.

Proof of Theorem 4.4. We consider two cases, $\alpha_{1} \neq 0$ and $\alpha_{1}=0$.

First, for $\alpha_{1} \neq 0$ (the force control is present in (1.1)), the exponential stability of $e^{t A^{\omega *}}$ has been established in [20] by using the multiplier method. Second, if $\alpha_{1}=0$ (only the moment control is applied), then $w_{1}$ is omitted everywhere; for instance, the state space of the subsystem (3.1) is $\mathscr{H}_{0}=H_{0}^{2} \times L^{2}(0, l) \times \mathbb{R}^{n_{2}}$ equipped with the inner product (3.4) with omission of $w_{1}$, that is, $\left\langle\left(y, z, w_{2}\right),\left(\tilde{y}, \tilde{z}, \tilde{w}_{2}\right)\right\rangle_{\mathscr{H}_{0}}=\int_{0}^{l}\left(E I y_{x x} \tilde{y}_{x x}-\rho \omega_{*}^{2} y \tilde{y}+\rho z \tilde{z}\right) d x+$ $2 \alpha_{2} \tilde{w}_{2}^{T} P_{2} w_{2}$, and the operator $A^{\omega_{*}}($ see $(3.2)-(3.3))$ is denoted by $A_{0}^{\omega_{*}}$, that is,

$$
\begin{aligned}
& \mathscr{D}\left(A_{0}^{\omega_{*}}\right)=\left\{\left(y, z, w_{2}\right) \in H_{0}^{4} \times H_{0}^{2} \times \mathbb{R}^{n_{2}} ;\right. \\
&\left.y_{x x x}(l)=0 ; E I y_{x x}(l)+\alpha_{2}\left[c_{2}^{T} w_{2}+d_{2} z_{x}(l)\right]=0\right\}, \\
& A_{0}^{\omega_{*}}\left(y, z, w_{2}\right)=\left(z,-\frac{E I}{\rho} y_{x x x x}+\omega_{*}^{2} y, A_{2} w_{2}+b_{2} z_{x}(l)\right) \quad \forall\left(y, z, w_{2}\right) \in \mathscr{D}\left(A_{0}^{\omega_{*}}\right) .
\end{aligned}
$$

Note that the coefficients $d_{2}, \gamma_{2}$ are positive by means of the assumption of Theorem 4.4 and hypothesis (H.III). Our goal is to show the uniform stability of the semigroup $e^{t A_{0}^{\omega *}}$. To do so, we have tried to use the multiplier technique without much success. However, one will use Huang's result [11] which corresponds to the frequency domain method. For this, consider the operator $A_{0}=A_{0}^{\omega_{*}}-\omega_{*}^{2} K$ with $\mathscr{D}\left(A_{0}\right)=\mathscr{D}\left(A_{0}^{\omega_{*}}\right)$, and $K$ is an operator on $\mathcal{H}_{0}$ defined as follows:

$$
K\left(y, z, w_{2}\right)=(0, y, 0) \text { for any }\left(y, z, w_{2}\right) \in \mathscr{H}_{0} .
$$

Obviously, the operator $K$ is compact on $\mathscr{H}_{0}$ and the operator $A_{0}$ satisfies all the properties of $A_{0}^{\omega_{*}}$, particularly Lemmas 3.1 and 4.1 and Proposition 4.2. Hence, $A_{0}$ generates a strongly stable semigroup of contractions denoted by $e^{t A_{0}}$. This leads us to claim that if the semigroup $e^{t A_{0}}$ is uniformly stable, then so is the semigroup $e^{t A_{0}^{\omega *}}$ [22]. In return, as has already been mentioned, $e^{t A_{0}}$ is a strongly stable semigroup of contractions, and hence, in order to obtain its uniform stability, we only have to show (see [11, Theorem 3, page 51]) that

$$
\sup \left\{\left\|\left(i \mu-A_{0}\right)^{-1}\right\|_{\mathscr{L}\left(\mathscr{H}_{0}\right)} ; \mu \in \mathbb{R}\right\}<\infty
$$


where $\|\cdot\| \mathscr{L}\left(\mathscr{H}_{0}\right)$ is the operator norm. For simplicity and without loss of generality, we assume that $E I=\rho=l=\alpha_{2}=1$. Consider then the resolvent equation, that is, given $\mu \in$ $\mathbb{R}$ and $(f, g, \xi) \in \mathscr{H}_{0}$, we seek $\left(y, z, w_{2}\right) \in \mathscr{D}\left(A_{0}\right)$ such that $\left(i \mu I-A_{0}\right)\left(y, z, w_{2}\right)=(f, g, \xi)$. Note that the resolvent estimate (4.17) can be derived as a consequence of the existence of a positive constant $M$, independent of $\mu$, such that

$$
\int_{0}^{1}\left(\left|y_{x x}\right|^{2}+|z|^{2}\right) d x+2\left|\bar{w}_{2}^{T} P_{2} w_{2}\right| \leq M\left(\left\|f_{x x}\right\|_{L^{2}(0, l)}^{2}+\|g\|_{L^{2}(0,1)}^{2}+|\xi|\right)
$$

which immediately gives (4.17). Using the known result of continuity of the function $\lambda \mapsto\left\|\left(\lambda-A_{0}\right)^{-1}\right\|$ for any $\lambda \in \rho\left(A_{0}\right)[21]$, it suffices to establish the estimate (4.17) for $|\mu|$ large. The proof, inspired by the work of Chen et al. [5] (see also [6]), is divided into 3 steps. Hereafter, $\|\cdot\|_{L^{2}(0,1)}$ is denoted by $\|\cdot\|$.

Step 1. The aim is to estimate $\left\|y_{x x}\right\|$, namely, to prove that for $\eta$ large,

$$
\left\|y_{x x}\right\| \leq M_{1}\left(\left\|f_{x x}\right\|+\|g\|+|\xi|\right)
$$

for some positive constant $M_{1}$. To accomplish this, let $\mu=\eta^{2}$, where $\eta \in \mathbb{R}$ (the estimates for $\mu=-\eta^{2}$ are similar). Thus the resolvent equation yields

$$
\begin{gathered}
y_{x x x x}-\eta^{4} y=i \eta^{2} f+g, \\
y_{x x}(1)+i \eta^{2} G_{2}\left(i \eta^{2}\right) y_{x}(1)-G_{2}\left(i \eta^{2}\right) f_{x}(1)+c_{2}^{T}\left(i \eta^{2} I-A_{2}\right)^{-1} \xi=0, \\
y(0)=y_{x}(0)=y_{x x x}(1)=0, \\
z=i \eta^{2} y-f, \\
w_{2}=i \eta^{2}\left(i \eta^{2} I-A_{2}\right)^{-1} b_{2} y_{x}(1)-\left(i \eta^{2} I-A_{2}\right)^{-1} b_{2} f_{x}(1)+\left(i \eta^{2} I-A_{2}\right)^{-1} \xi,
\end{gathered}
$$

where $G_{2}(\cdot)$ is given by $(2.3)$. Consider now the following two systems of linear differential equations:

$$
\begin{gathered}
\hat{y}_{x x x x}-\eta^{4} \hat{y}=i \eta^{2} f+g, \\
\hat{y}(0)=\hat{y}_{x}(0)=\hat{y}_{x x}(0)=\hat{y}_{x x x}(0)=0, \\
\tilde{y}_{x x x x}-\eta^{4} \tilde{y}=0, \\
\tilde{y}(0)=\tilde{y}_{x}(0)=0, \\
\tilde{y}_{x x}(1)+i \eta^{2} G_{2}\left(i \eta^{2}\right) \tilde{y}_{x}(1)=r_{1}, \\
-\tilde{y}_{x x x}(1)=r_{2},
\end{gathered}
$$

where

$$
\begin{gathered}
r_{1}=-i \eta^{2} G_{2}\left(i \eta^{2}\right) \hat{y}_{x}(1)-\hat{y}_{x x}(1)+G_{2}\left(i \eta^{2}\right) f_{x}(1)-c_{2}^{T}\left(i \eta^{2} I-A_{2}\right)^{-1} \xi, \\
r_{2}=\hat{y}_{x x x}(1) .
\end{gathered}
$$


Clearly, if $\hat{y}(x)$ and $\tilde{y}(x)$ are the solutions of (4.21) and (4.22), respectively, then $y(x)=$ $\hat{y}(x)+\tilde{y}(x)$ satisfies (4.20). Furthermore, the unique solution of (4.21) is

$$
\hat{y}(x)=\frac{1}{2} \int_{0}^{x} \eta^{-3}[\sinh (\eta(x-\tau))-\sin (\eta(x-\tau))]\left(i \eta^{2} f(\tau)+g(\tau)\right) d \tau,
$$

whereas the general solution of (4.22) is given by

$$
\tilde{y}(x)=W e^{\eta x}+X e^{i \eta x}+Y e^{-\eta x}+Z e^{-i \eta x} .
$$

Here $W, X, Y$, and $Z$ are to be determined from the boundary conditions of (4.22) which lead us to a linear system $M\left(\begin{array}{llll}W & X & Y & Z\end{array}\right)^{T}=\left(\begin{array}{llll}0 & 0 & r_{1} & r_{2}\end{array}\right)^{T}$, where the superscript $T$ stands for the transpose and $M=\left(m_{i j}\right)_{1 \leq i, j \leq 4}$ is a matrix whose elements are

$$
\begin{aligned}
& m_{11}=1, \quad m_{12}=1, \quad m_{13}=1, \quad m_{14}=1, \quad m_{21}=1, \quad m_{22}=i, \quad m_{23}=-1, \quad m_{24}=-i, \\
& m_{31}=\left[\eta^{2}+i \eta^{3} G_{2\left(i \eta^{2}\right)}\right] e^{\eta}, \quad m_{32}=-\left[\eta^{2}+\eta^{3} G_{2\left(i \eta^{2}\right)}\right] e^{i \eta}, \quad m_{33}=\left[\eta^{2}-i \eta^{3} G_{2\left(i \eta^{2}\right)}\right] e^{-\eta}, \\
& m_{34}=\left[\eta^{3} G_{2\left(i \eta^{2}\right)}-\eta^{2}\right] e^{-i \eta}, \quad m_{41}=-\eta^{3} e^{\eta}, \quad m_{42}=i \eta^{3} e^{i \eta}, \quad m_{43}=\eta^{3} e^{-\eta}, \quad m_{44}=-i \eta^{3} e^{-i \eta} .
\end{aligned}
$$

Note that for $\eta$ large, $\operatorname{det} M \neq 0$ (see (4.37)), and hence

$$
\left(\begin{array}{c}
W \\
X \\
Y \\
Z
\end{array}\right)=(i+1) \eta^{2}(\operatorname{det} M)^{-1}\left(\begin{array}{cccc}
\times & \times & \mu_{13} & -\mu_{14} \\
\times & \times & -\mu_{23} & -\mu_{24} \\
\times & \times & -\mu_{33} & \mu_{34} \\
\times & \times & -\mu_{43} & -\mu_{44}
\end{array}\right)\left(\begin{array}{c}
0 \\
0 \\
r_{1} \\
r_{2}
\end{array}\right)
$$

where $\times$ denote unnecessary elements for subsequent calculations and

$$
\begin{aligned}
& \mu_{13}=\eta e^{i \eta}+i \eta e^{-i \eta}+(i+1) \eta e^{-\eta}, \quad \mu_{23}=i \eta e^{\eta}+(i+1) \eta e^{-i \eta}+\eta e^{-\eta}, \\
& \mu_{14}=i\left[1+\eta G_{2}\left(i \eta^{2}\right)\right] e^{i \eta}+\left[1-\eta G_{2}\left(i \eta^{2}\right)\right] e^{-i \eta}+\left[i+1+(1-i) \eta G_{2}\left(i \eta^{2}\right)\right] e^{-\eta}, \\
& \mu_{24}=\left[i-\eta G_{2}\left(i \eta^{2}\right)\right] e^{\eta}+(i-1)\left[1-\eta G_{2}\left(i \eta^{2}\right)\right] e^{-i \eta}+\left[i \eta G_{2}\left(i \eta^{2}\right)-1\right] e^{-\eta}, \\
& \mu_{33}=-i \eta e^{i \eta}-(i+1) \eta e^{\eta}-\eta e^{-i \eta}, \quad \mu_{43}=(i+1) \eta e^{i \eta}+\eta e^{\eta}+i \eta e^{-\eta}, \\
& \mu_{34}=\left[1+\eta G_{2}\left(i \eta^{2}\right)\right] e^{i \eta}+\left[(i+1)+(i-1) \eta G_{2}\left(i \eta^{2}\right)\right] e^{\eta}+i\left[1-\eta G_{2}\left(i \eta^{2}\right)\right] e^{-i \eta}, \\
& \mu_{44}=(1-i)\left[1+\eta G_{2}\left(i \eta^{2}\right)\right] e^{i \eta}+\left[1+i \eta G_{2}\left(i \eta^{2}\right)\right] e^{\eta}-\left[i+\eta G_{2}\left(i \eta^{2}\right)\right] e^{-\eta} .
\end{aligned}
$$

After differentiating and using integration by parts twice in (4.24), we get

$$
\begin{aligned}
& \hat{y}_{x x}(x)=\frac{\eta^{-1} e^{\eta x}}{4} \int_{0}^{1} e^{-\eta \tau}\left[i f_{\tau \tau}(\tau)+g(\tau)\right] d \tau+\mathcal{O}\left(\eta^{-1}\left[\left\|f_{x x}\right\|+\|g\|\right]\right), \\
& \hat{y}_{x}(x)=-\frac{i}{\eta^{2}} f_{x}(x)+\frac{\eta^{-2}}{4} \int_{0}^{1} e^{\eta(x-\tau)}\left[i f_{\tau \tau}(\tau)+g(\tau)\right] d \tau+\mathcal{O}\left(\eta^{-2}\left[\left\|f_{x x}\right\|+\|g\|\right]\right), \\
& \hat{y}_{x x x}(x)=\frac{1}{4} \int_{0}^{1} e^{\eta(x-\tau)}\left[i f_{\tau \tau}(\tau)+g(\tau)\right] d \tau+\mathcal{O}\left(\left\|f_{x x}\right\|+\|g\|\right) .
\end{aligned}
$$


Combining (4.23) and (4.29) and using (2.5)-(2.6) yield

$$
\begin{aligned}
& r_{1}=-\frac{e^{\eta}}{4}\left[i G_{2}\left(i \eta^{2}\right)+\eta^{-1}\right] \int_{0}^{1} e^{-\eta \tau}\left(i f_{\tau \tau}(\tau)+g(\tau)\right) d \tau+\mathcal{O}\left(\left\|f_{x x}\right\|+\|g\|\right)+\mathcal{O}\left(\eta^{-2}|\xi|\right), \\
& r_{2}=\frac{e^{\eta}}{4} \int_{0}^{1} e^{-\eta \tau}\left(i f_{\tau \tau}(\tau)+g(\tau)\right) d \tau+\mathcal{O}\left(\left\|f_{x x}\right\|+\|g\|\right) .
\end{aligned}
$$

We now define $\Delta$ by

$$
\begin{aligned}
\Delta & =-(i+1) \eta^{2}\left\{\left[i G_{2}\left(i \eta^{2}\right)+\eta^{-1}\right] \mu_{13}+\mu_{14}\right\} \\
& =(i+1)\left\{-e^{i \eta}\left[2 i \eta^{3} G_{2}\left(i \eta^{2}\right)+(i+1) \eta^{2}\right]+e^{-i \eta}\left[2 \eta^{3} G_{2}\left(i \eta^{2}\right)-(i+1) \eta^{2}\right]-2(i+1) \eta^{2} e^{-\eta}\right\} .
\end{aligned}
$$

From the properties of the transfer function $G_{2}(\cdot)$ cited in (2.5) and (2.6), it follows that the dominant term of $\Delta$ is $\eta^{3}$, that is,

$$
\Delta=O\left(\eta^{3}\right)
$$

Moreover, using the known inequality $|a+b| \geq|a|-|b|$ for (4.32) yields

$$
|\Delta| \geq \sqrt{2}\left[\left|2 i \eta^{3} G_{2}\left(i \eta^{2}\right)+(i+1) \eta^{2}\right|-\left|2 \eta^{3} G_{2}\left(i \eta^{2}\right)-(i+1) \eta^{2}\right|\right]
$$

for $\eta$ large. Combine now (2.5), (2.6), and (4.34). As a result, we obtain after a straightforward calculation,

$$
|\Delta| \geq M\left(\gamma_{2}\right) \eta^{2}
$$

for $\eta$ sufficiently large and for a positive constant $M$ depending on $\gamma_{2}$. Furthermore, it follows from the definition of the matrix $M$ that

$$
\begin{aligned}
\operatorname{det} M= & -\eta^{3} e^{\eta}\left(\Delta+2(i+1) \eta^{2} e^{-\eta}\right)-2 \eta^{4} e^{(i-1) \eta}\left[-(i+1) \eta^{2} G_{2}\left(i \eta^{2}\right)-i \eta\right] \\
& -2 \eta^{4} e^{-(i+1) \eta}\left[(i-1) \eta^{2} G_{2}\left(i \eta^{2}\right)-i \eta\right]+8 i \eta^{5}
\end{aligned}
$$

where $\Delta$ is defined in (4.32). Except for the first term $-\eta^{3} e^{\eta} \Delta$ of (4.36), all the others are bounded by $O\left(\eta^{5}\right)$ for $\eta$ sufficiently large. Consequently,

$$
\operatorname{det} M=-\eta^{3} e^{\eta} \Delta+O\left(\eta^{5}\right)
$$

which implies, by (4.35), that

$$
(\operatorname{det} M)^{-1}=-\eta^{-3} e^{-\eta}(\Delta)^{-1}+\mathcal{O}\left(\eta^{-5} e^{-2 \eta}\right)=\mathcal{O}\left(\eta^{-5} e^{-\eta}\right) .
$$

We now estimate $W$ (see (4.25) and (4.27)). Clearly, system (4.27) gives $W=(i+$ 1) $\eta^{2}(\operatorname{det} M)^{-1}\left(\mu_{13} r_{1}-\mu_{14} r_{2}\right)$. Combining (4.30)-(4.32) and the first estimate of (4.38), 
we deduce that

$$
\begin{aligned}
W= & -\frac{1}{4 \eta^{3}} \int_{0}^{1} e^{-\eta \tau}\left[i f_{\tau \tau}(\tau)+g(\tau)\right] d \tau+\frac{e^{\eta} \Delta}{4} \mathcal{O}\left(\eta^{-5} e^{-2 \eta}\right) \int_{0}^{1} e^{-\eta \tau}\left[i f_{\tau \tau}(\tau)+g(\tau)\right] d \tau \\
& +(i+1) \eta^{2}(\operatorname{det} M)^{-1}\left\{\mu_{13}\left[\mathcal{O}\left(\left\|f_{x x}\right\|+\|g\|\right)+\mathcal{O}\left(\eta^{-2}|\xi|\right)\right]-\mu_{14} \mathcal{O}\left(\left\|f_{x x}\right\|+\|g\|\right)\right\} .
\end{aligned}
$$

But $\mu_{13}$ and $\mu_{14}$ (see (4.27)) are bounded by $O(\eta)$. This, together with (4.33) and the second estimate of (4.38), implies that (4.39) can be written as follows:

$$
W=-\frac{1}{4 \eta^{3}} \int_{0}^{1} e^{-\eta \tau}\left[i f_{\tau \tau}(\tau)+g(\tau)\right] d \tau+\mathcal{O}\left(\eta^{-2} e^{-\eta}\left[|| f_{x x}\|+\| g \|\right]\right)+\mathcal{O}\left(\eta^{-5} e^{-\eta}|\xi|\right)
$$

Now, our aim is to derive estimates of $X, Y$, and $Z$. Using once again (4.27), we have $X=-(i+1) \eta^{2}(\operatorname{det} M)^{-1}\left(\mu_{23} r_{1}+\mu_{24} r_{2}\right)$. Then, one can show from (4.30), (4.31), and the expressions of $\mu_{23}, \mu_{24}$ that the coefficient of the dominant term $e^{2 \eta}$, appearing in $\mu_{23} r_{1}+$ $\mu_{24} r_{2}$, is zero. Indeed, we have

$$
\begin{aligned}
& X=(\operatorname{det} M)^{-1} O\left(\eta^{3} e^{\eta}\left[\left\|f_{x x}\right\|+\|g\|\right]\right)+\mathcal{O}\left(\eta e^{\eta}|\xi|\right) \\
& =\mathcal{O}\left(\eta^{-2}\left[\left\|f_{x x}||+\right\| g \|\right]\right)+\mathcal{O}\left(\eta^{-4}|\xi|\right),
\end{aligned}
$$

where the second estimate is obtained by means of (4.38). Similarly,

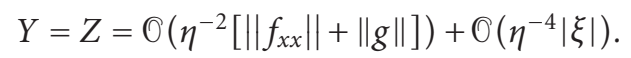

Now, we are ready to estimate $\left\|y_{x x}\right\|$. Recall first that, by construction, $y(x)=\hat{y}(x)+$ $\tilde{y}(x)$, where $\hat{y}(x)$ and $\tilde{y}(x)$ are given by (4.24) and (4.25), respectively. Then it follows from (4.29) and (4.40)-(4.42) that

$$
\begin{aligned}
\left\|y_{x x}\right\|= & \left\{\mathcal{O}\left(e^{-\eta}\left[\left\|f_{x x}\right\|+\|g\|\right]\right)+\mathcal{O}\left(\eta^{-3} e^{-\eta}|\xi|\right)\right\}\left(\int_{0}^{1} e^{2 \eta x} d x\right)^{1 / 2} \\
& +\left\{\mathcal{O}\left(\left\|f_{x x}\right\|+\|g\|\right)+\mathcal{O}\left(\eta^{-2}|\xi|\right)\right\}\left(\int_{0}^{1} e^{-2 \eta x} d x\right)^{1 / 2} \\
& +\mathcal{O}\left(\left\|f_{x x}\right\|+\|g\|\right)+\mathcal{O}\left(\eta^{-2}|\xi|\right) \\
= & \mathcal{O}\left(\left\|f_{x x}\right\|+\|g\|\right)+\mathcal{O}\left(\eta^{-2}|\xi|\right) .
\end{aligned}
$$

As a result, we arrived at the desired estimate (4.19) of Step 1.

Step 2. The goal is to derive an estimate of $\|z\|$, where $z=i \eta^{2} y-f$ (see (4.20)), that is, $\|z\| \leq M_{2}\left(\left\|f_{x x}\right\|+\|g\|+|\xi|\right)$ for a positive constant $M_{2}$. In return, $\|z\| \leq \eta^{2}\|y\|+\|f\|$. Thus, it suffices to estimate $\eta^{2}\|y\|$. To this end, one can show in a similar way as for the estimate (4.29) that

$$
\hat{y}(x)=-\frac{i}{\eta^{2}} f(x)+\frac{\eta^{-3}}{4} \int_{0}^{1} e^{\eta(x-\tau)}\left(i f_{\tau \tau}(\tau)+g(\tau)\right) d \tau+O\left(\eta^{-3}\left[\left\|f_{x x}\right\|+\|g\|\right]\right) .
$$


Combining (4.25), (4.40)-(4.42), and (4.44), we obtain

$$
\begin{aligned}
\|y\|= & \eta^{-2}\|f\|+O\left(\eta^{-3}\left[\left\|f_{x x}\right\|+\|g\|\right]\right) \\
& +\left\{\mathcal{O}\left(\eta^{-2} e^{-\eta}\left[\left\|f_{x x}\right\|+\|g\|\right]\right)+\mathcal{O}\left(\eta^{-5} e^{-\eta}|\xi|\right)\right\}\left(\int_{0}^{1} e^{2 \eta x} d x\right)^{1 / 2} \\
& +\left\{\mathcal{O}\left(\eta^{-2}\left[\left\|f_{x x}\right\|+\|g\|\right]\right)+\mathcal{O}\left(\eta^{-4}|\xi|\right)\right\}\left(\int_{0}^{1} e^{-2 \eta x} d x\right)^{1 / 2} \\
& +\mathcal{O}\left(\eta^{-2}\left[\left\|f_{x x}\right\|+\|g\|\right]\right)+\mathcal{O}\left(\eta^{-4}|\xi|\right),
\end{aligned}
$$

which implies that $\eta^{2}\|y\|=\mathscr{O}\left(\left\|f_{x x}\right\|+\|g\|\right)+\mathcal{O}\left(\eta^{-2}|\xi|\right)$. Thus

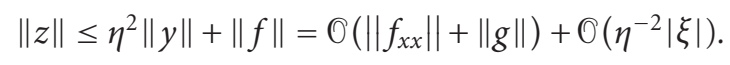

This achieves Step 2.

Step 3. All we need to do is to establish that $\left|w_{2}\right| \leq M_{3}\left(\left\|f_{x x}\right\|+\|g\|+|\xi|\right)$ for a positive constant $M_{3}$, where $w_{2}$ is defined in (4.20). This immediately yields

$$
\begin{aligned}
\left|w_{2}\right| & =\mathscr{O}\left(\left|y_{x}(1)\right|\left|b_{2}\right|\right)+\mathcal{O}\left(\eta^{-2}|| f_{x x}||\left|b_{2}\right|\right)+\mathcal{O}\left(\eta^{-2}|\xi|\right) \\
& =\mathscr{O}\left(\left|y_{x}(1)\right|\right)+\mathcal{O}\left(\eta^{-2}|| f_{x x}||\right)+\mathcal{O}\left(\eta^{-2}|\xi|\right) .
\end{aligned}
$$

Using the second estimate of (4.29) and (4.40), (4.41), and (4.42) and arguing in the same way as for (4.43), we get

$$
\begin{aligned}
& \left|y_{x}(1)\right|=\mid-\frac{i}{\eta^{2}} f_{x}(1)+\frac{e^{\eta}}{4 \eta^{2}} \int_{0}^{1} e^{-\eta \tau}\left(i f_{\tau \tau}(\tau)+g(\tau)\right) d \tau+\mathcal{O}\left(\eta^{-2}\left[\left\|f_{x x}\right\|+\|g\|\right]\right) \\
& +\eta W e^{\eta}+i \eta X e^{i \eta}-\eta Y e^{-\eta}-i \eta Z e^{-i \eta x} \\
& =O\left(\eta^{-1}\left[\left\|f_{x x}\right\|+\|g\|\right]\right)+O\left(\eta^{-3}|\xi|\right) \text {. }
\end{aligned}
$$

This, together with (4.47), gives

$$
\left|w_{2}\right|=O\left(\eta^{-1}\left[\left\|f_{x x}\right\|+\|g\|\right]\right)+O\left(\eta^{-2}|\xi|\right) .
$$

After all these three steps, the desired estimate (4.18) follows easily. The proof of Theorem 4.4 is complete.

\section{Stability of the global system}

Proof of Theorem 2.2. Recall first that the solution $\Phi(t)$ of the global system (2.11) (see also (3.7)) stemmed from $\Phi_{0}=\left(\phi_{0}, \omega_{0}\right) \in \mathscr{D}(\mathscr{A})$ can be written as $\Phi(t)=(\phi(t), \omega(t))$, where $\phi(t)=\left(y(\cdot, t), y_{t}(\cdot, t), w_{1}(t), w_{2}(t)\right)$ is the unique solution of the subsystem $(3.1)$ perturbed by the operator $\left(\omega^{2}-\omega_{*}^{2}\right) K$ (see $\left.(4.16)\right)$, that is,

$$
\phi_{t}(t)=\left[A^{\omega_{*}}+\left(\omega^{2}(t)-\omega_{*}^{2}\right) K\right] \phi(t)
$$


and $\omega(t)$ is solution of the ordinary differential equation

$$
\dot{\omega}(t)=\frac{-\gamma\left(\omega-\omega_{*}\right)-2 \rho \omega(t)\left\langle y, y_{t}\right\rangle_{L^{2}(0, l)}}{I_{d}+\rho\|y\|_{L^{2}(0, l)}^{2}} .
$$

Furthermore, the Lyapunov function $\mathscr{L}$ (see (3.8)) is nonincreasing. Hence, the integral $\int_{0}^{+\infty}\left(\omega(t)-\omega_{*}\right)^{2} d t$ converges and the solution $(\phi(t), \omega(t))$ is bounded in $\mathscr{X}$. This implies, thanks to (5.2), that the function $\omega(t)-\omega_{*}$ and its derivative $(d / d t)\left(\omega(t)-\omega_{*}\right)$ are bounded. Consequently, using Barbalat's lemma [14], the three properties of $\omega(t)-\omega_{*}$ cited above lead us to claim that $\lim _{t \rightarrow+\infty} \omega(t)=\omega_{*}$. Thus, for all $\epsilon>0$, there exists $\tau$ sufficiently large such that for any $t \geq \tau$,

$$
\left|\omega^{2}(t)-\omega_{*}^{2}\right|<\epsilon
$$

As mentioned in Theorem 4.4, the subsystem (3.1) is exponentially stable, and therefore there exist (uniform) constants $\tilde{M}, \tilde{\mu}>0$ such that

$$
\left\|e^{t A^{\omega *}}\right\|_{\mathscr{L}(\mathscr{H})} \leq \tilde{M} e^{-\tilde{\mu} t} \quad \forall t \geq 0,
$$

where $\|\cdot\|_{\mathscr{L}(\mathscr{H})}$ is the operator norm. Now, we return to the subsystem (5.1). Given a positive real number $\tau$, the solution of (5.1) is given by

$$
\phi(t)=e^{(t-\tau) A^{\omega *}} \phi(\tau)+\int_{\tau}^{t} e^{(t-s) A^{\omega *}}\left(\omega^{2}(s)-\omega_{*}^{2}\right) K \phi(s) d s
$$

for any $t \geq \tau$. This, together with (5.3) and (5.4), implies that

$$
\|\phi(t)\|_{\mathscr{H}} \leq \tilde{M} e^{-\tilde{\mu}(t-\tau)}\|\phi(\tau)\|_{\mathscr{H}}+\epsilon \tilde{M} \int_{\tau}^{t} e^{-\tilde{\mu}(t-\tau)}\|\phi(s)\|_{\mathscr{H}} d s .
$$

Therefore,

$$
\left\|e^{\tilde{\mu} t} \phi(t)\right\|_{\mathscr{H}} \leq \tilde{M}\left\|e^{\tilde{\mu} \tau} \phi(\tau)\right\|_{\mathscr{H}}+\left.\epsilon \tilde{M} \int_{\tau}^{t}\left\|e^{\tilde{\mu} s} \phi(s)\right\|\right|_{\mathscr{H}} d s .
$$

Using the fact that $\phi(t)$ is bounded, one can apply Gronwall's lemma to (5.7) to get

$$
\|\phi(t)\|_{\mathscr{H}} \leq \tilde{M}\|\phi(\tau)\|_{\mathscr{H}} e^{-(\tilde{\mu}-\epsilon \tilde{M})(t-\tau)}
$$

for any $t \geq \tau$. Now, we choose $\epsilon$ so that $\mu-\epsilon \tilde{M}>0$, and hence $\phi(t)$ is exponentially stable in $\mathcal{H}$. Finally, returning to the differential equation (5.2), one proves analogously to [24] that $\omega-\omega_{*} \rightarrow 0$ exponentially in $\mathbb{R}$. Indeed, (5.2) implies that

$$
\begin{aligned}
\left|e^{\gamma t / I_{d}}\left(\omega(t)-\omega_{*}\right)\right| \leq & e^{\gamma \tau / I_{d}}\left|\omega(\tau)-\omega_{*}\right| \\
& +\int_{\tau}^{t} e^{\gamma s / I_{d}}\left[\frac{\gamma \rho}{I_{d}{ }^{2}}\left|\omega(s)-\omega_{*}\right|+\left.\frac{2 \rho}{I_{d}}|\omega(s)|\|y(s)\|\right|_{L^{2}}\left\|y_{t}(s)\right\|_{L^{2}}\right] d s .
\end{aligned}
$$


Using the definition of the norm of $\phi(t)$ and (5.8), we have

$$
\|y(s)\|_{L^{2}}\left\|y_{t}(s)\right\|_{L^{2}} \leq\|\phi(s)\|_{\mathscr{H}}^{2} \leq\left(\tilde{M}\|\phi(\tau)\|_{\mathscr{H}}\right)^{2} e^{-2(\tilde{\mu}-\epsilon \tilde{M})(s-\tau)} .
$$

It remains to substitute (5.10) into (5.9) and use Gronwall's lemma to obtain the exponential stability of $\omega(t)-\omega_{*}$. This achieves the proof of Theorem 2.2.

Remark 5.1. The decay rate obtained in Theorem 2.2, although exponential, is not uniform. This is due to the fact that the constants of the decay rate depend on the initial condition $\Phi_{0}=\left(\phi_{0}, \omega_{0}\right)$.

\section{Conclusion}

In this paper, we have proposed a feedback law which stabilizes a body-beam system in the case where the rigid body is rotating with a nonconstant angular velocity. We have shown that if the angular velocity is smaller than $\left(1 / l^{2}\right) \sqrt{12 E I / \rho}$, the system is exponentially stable as soon as a control torque is applied to the rigid body and either a dynamic boundary control moment or a dynamic boundary control force or both of them act on the free end of the beam. This result improves those obtained in [20] for nonconstant angular velocity and in [16] (static feedback case) for dynamic controls.

An interesting research problem would be the extension of the results presented in this paper to nonlinear dynamic controls. This question is motivated by the fact that, in practice, the input amplitudes are constrained by the power of the actuators which go into nonlinear saturations [1]. Therefore, the stability of such systems should be assured with nonlinear controls. This will be the subject of a forthcoming paper.

\section{Appendix}

Proof of (4.13). It is easy to see that $\lambda_{n}$ is an eigenvalue of the operator $B_{0}$ if and only if there is a nontrivial element $v_{n} \in \mathscr{D}\left(B_{0}\right)$ satisfying the following system:

$$
\begin{gathered}
v_{n_{x x x x}}-\sigma_{n}{ }^{4} v_{n}=0, \\
v(0)=v_{n_{x}}(0)=0, \\
v_{n_{x x x}}(1)=v_{n_{x x}}(1)=0,
\end{gathered}
$$

where

$$
\sigma_{n}^{4}=\lambda_{n}+\omega_{*}^{2}
$$

The general solution of (A.1)-(A.2) is

$$
v_{n}=C_{1}\left(\cosh \sigma_{n} x-\cos \sigma_{n} x\right)+C_{2}\left(\sinh \sigma_{n} x-\sin \sigma_{n} x\right),
$$

where $C_{1}$ and $C_{2}$ are to be determined by means of (A.3), that is,

$$
\left(\begin{array}{cc}
\sinh \sigma_{n} l-\sin \sigma_{n} & \cosh \sigma_{n}+\cos \sigma_{n} \\
\cosh \sigma_{n} l+\cos \sigma_{n} & \sinh \sigma_{n}+\sin \sigma_{n}
\end{array}\right)\left(\begin{array}{l}
C_{1} \\
C_{2}
\end{array}\right)=\left(\begin{array}{l}
0 \\
0
\end{array}\right)
$$


Thus, $v_{n}$ is an eigenfunction of $B_{0}$ if

$$
\cosh \sigma_{n} \cos \sigma_{n}+1=0 .
$$

Using the results of Langer [15], one can check that the asymptotic estimate of solu-

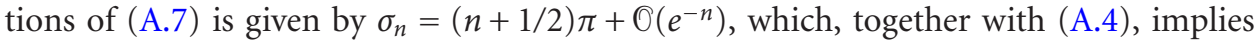
that $\lim _{n \rightarrow+\infty}\left|\sqrt{\lambda_{n+1}}-\sqrt{\lambda_{n}}\right|=\infty$. We prove now that each eigenfunction $v_{n}$ of $B_{0}$ satisfies $v_{n_{x}}(1) \neq 0, n=1,2, \ldots$ (the proof of $v_{n}(1) \neq 0$ is similar). Suppose the contrary is true, that is, $v_{n_{x}}(1)=0$, and therefore (A.5) yields $C_{1}\left(\sinh \sigma_{n}+\sin \sigma_{n}\right)+C_{2}\left(\cosh \sigma_{n}-\cos \sigma_{n}\right)=0$. Combining this last equation with system (A.6), one can show that $C_{1}=C_{2}=0$, which contradicts the fact that $v_{n}$ is an eigenfunction.

\section{Acknowledgment}

The author acknowledges the support of Sultan Qaboos University.

\section{References}

[1] J. Ackermann, Sampled-Data Control System: Analysis and Synthesis, Robust System Design, Springer-Verlag, Berlin, 1985.

[2] J. Baillieul and M. Levi, Rotational elastic dynamics, Phys. D 27 (1987), no. 1-2, 43-62.

[3] A. M. Bloch and E. S. Titi, On the dynamics of rotating elastic beams, New Trends in Systems Theory (Genoa, 1990) (G. Conte, A. Perdon, and B. Wyman, eds.), Progr. Systems Control Theory, vol. 7, Birkhäuser Boston, Massachusetts, 1991, pp. 128-135.

[4] H. Brezis, Analyse Fonctionnelle. Théorie et Applications, Collection Mathématiques Appliquées pour la Maîtrise, Masson, Paris, 1983.

[5] G. Chen, S. G. Krantz, D. W. Ma, C. E. Wayne, and H. H. West, The Euler-Bernoulli beam equation with boundary energy dissipation, Operator Methods for Optimal Control Problems (New Orleans, La, 1986) (S. J. Lee, ed.), Lecture Notes in Pure and Appl. Math., vol. 108, Marcel Dekker, New York, 1987, pp. 67-96.

[6] B. Chentouf, Boundary feedback stabilization of a variant of the SCOLE model, J. Dynam. Control Systems 9 (2003), no. 2, 201-232.

[7] B. Chentouf and J.-F. Couchouron, Nonlinear feedback stabilization of a rotating body-beam without damping, ESAIM Control Optim. Calc. Var. 4 (1999), 515-535.

[8] B. Chentouf, C. Z. Xu, and G. Sallet, On the stabilization of a vibrating equation, Nonlinear Anal. Ser. A: Theory and Methods 39 (2000), no. 5, 537-558.

[9] J.-M. Coron and B. d'Andréa Novel, Stabilization of a rotating body beam without damping, IEEE Trans. Automat. Control 43 (1998), no. 5, 608-618.

[10] A. Haraux, Systèmes Dynamiques Dissipatifs et Applications, Recherches en Mathématiques Appliquées, vol. 17, Masson, Paris, 1991.

[11] F. L. Huang, Characteristic conditions for exponential stability of linear dynamical systems in Hilbert spaces, Ann. Differential Equations 1 (1985), no. 1, 43-56.

[12] A. E. Ingham, Some trigonometrical inequalities with applications to the theory of series, Math. Z. 41 (1936), 367-379.

[13] T. Kato, Perturbation Theory for Linear Operators, Springer-Verlag, Berlin, 1976.

[14] H. K. Khalil, Nonlinear Systems, Prentice-Hall, New Jersey, 2002.

[15] R. E. Langer, On the zero of exponential sums and integrals, Bull. Amer. Math. Soc. (1931), 213239.

[16] H. Laousy, C. Z. Xu, and G. Sallet, Boundary feedback stabilization of a rotating body-beam system, IEEE Trans. Automat. Control 41 (1996), no. 2, 241-245. 
[17] Ö. Morgül, Constant angular velocity control of a rotating flexible structure, Proc. 2nd European Control Conference (Groningen, 1993), pp. 299-302.

[18] Orientation and stabilization of a flexible beam attached to a rigid body: planar motion, IEEE Trans. Automat. Control 36 (1991), no. 8, 953-962.

[19] Dynamic boundary control of an Euler-Bernoulli beam, IEEE Trans. Automat. Control 37 (1992), no. 5, 639-642.

[20] Control and stabilization of a rotating flexible structure, Automatica J. IFAC 30 (1994), no. 2, 351-356.

[21] A. Pazy, Semigroups of Linear Operators and Applications to Partial Differential Equations, Applied Mathematical Sciences, vol. 44, Springer-Verlag, New York, 1983.

[22] R. Triggiani, Lack of uniform stabilization for noncontractive semigroups under compact perturbation, Proc. Amer. Math. Soc. 105 (1989), no. 2, 375-383.

[23] M. Vidyasagar, Nonlinear Systems Analysis, Prentice-Hall, New Jersey, 1978.

[24] C.-Z. Xu and J. Baillieul, Stabilizability and stabilization of a rotating body-beam system with torque control, IEEE Trans. Automat. Control 38 (1993), no. 12, 1754-1765.

[25] C.-Z. Xu and G. Sallet, Boundary stabilization of rotating flexible systems, Analysis and Optimization of Systems: State and Frequency Domain Approaches for Infinite-Dimensional Systems (Sophia-Antipolis, 1992) (R. F. Curtain, A. Bensoussan, and J. L. Lions, eds.), Lecture Notes in Control and Inform. Sci., vol. 185, Springer-Verlag, Berlin, 1993, pp. 347-365.

Boumediène Chentouf: Department of Mathematics and Statistics, Sultan Qaboos University, P.O. Box 36, Al-Khod 123, Sultanate of Oman

E-mail address: chentouf@squ.edu.om 


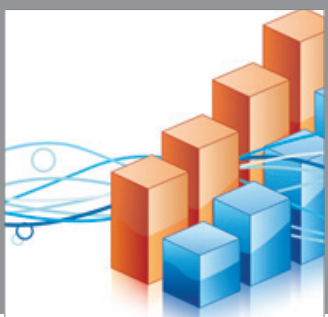

Advances in

Operations Research

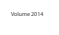

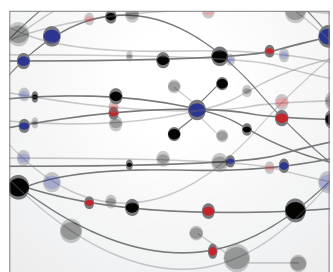

\section{The Scientific} World Journal
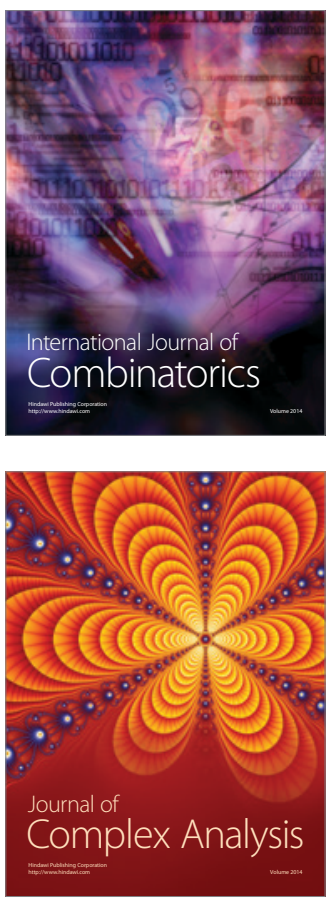

International Journal of

Mathematics and

Mathematical

Sciences
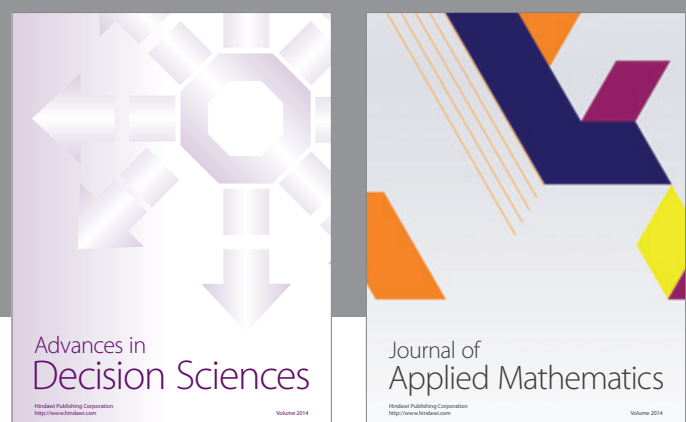

Journal of

Applied Mathematics
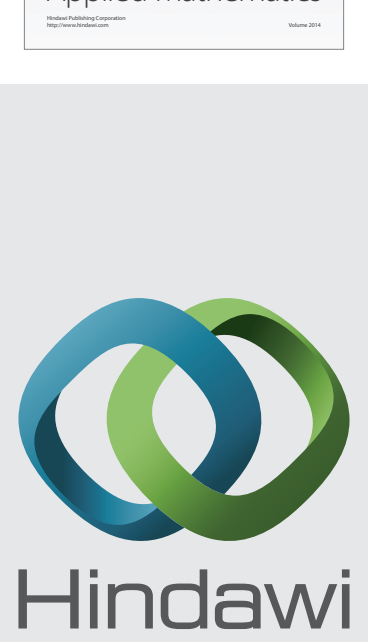

Submit your manuscripts at http://www.hindawi.com
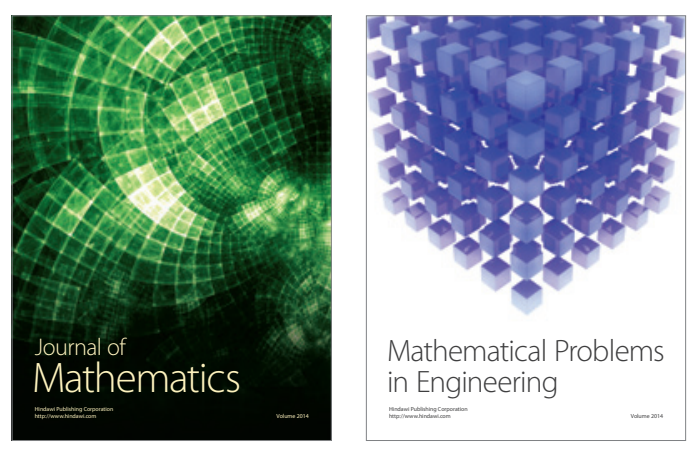

Mathematical Problems in Engineering
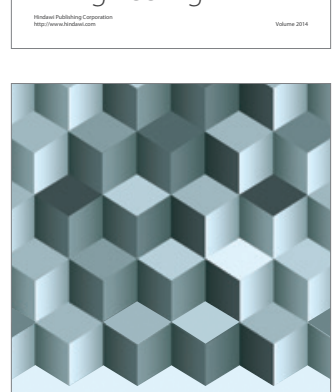

Journal of

Function Spaces
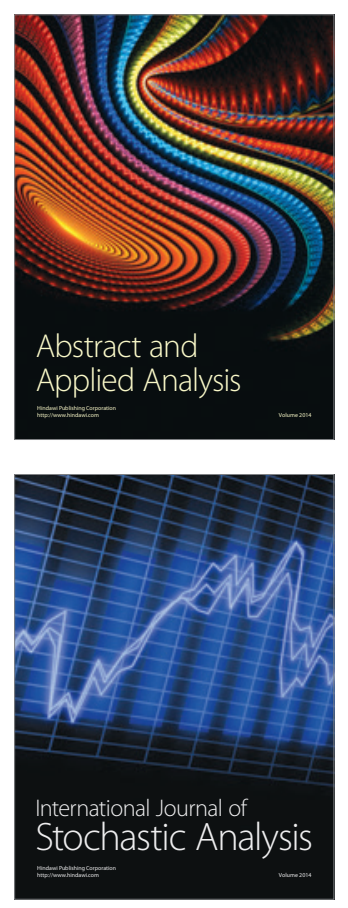

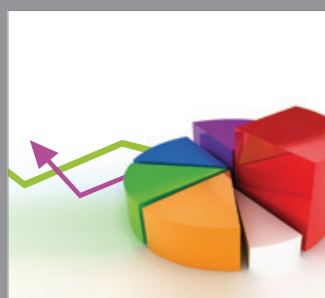

ournal of

Probability and Statistics

Promensencen
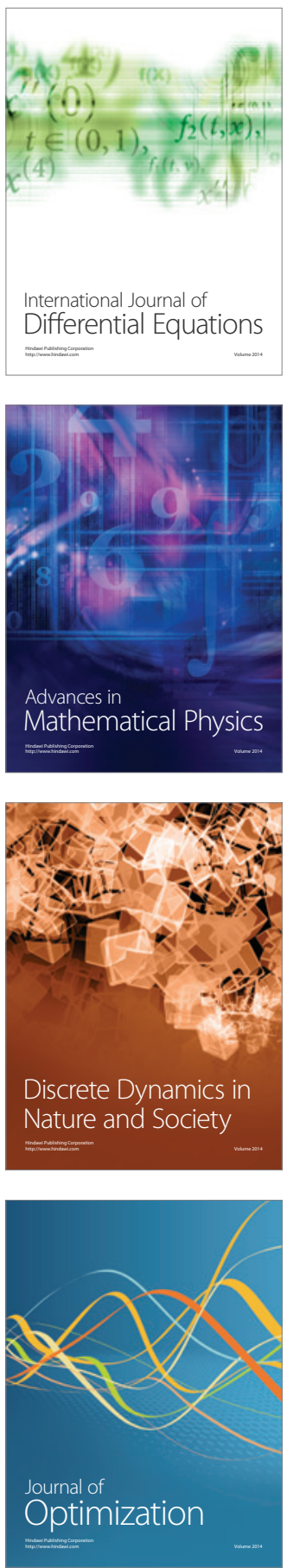